\title{
ARTERY FIRST TECHNIQUE FOR MANAGEMENT OF ABERRANT HEPATIC ARTERIAL ANATOMY DURING PANCREATICODUODENECTOMY - EXPERIENCE FROM A SPECIALIZED HEPATOPANCREATOBILIARY UNIT
}

\author{
Muhammad Taqi Pirzada, Raza Sayyed, Anam Muzaffar, Aamir Ali Syed, Faisal Hanif \\ Department of Surgical Oncology, Shaukat Khanum Memorial Cancer Hospital and Research Centre, Lahore, \\ Pakistan
}

Received: 2 February 2016 / Accepted: 16 July 2016

\begin{abstract}
Purpose: Aberrant hepatic arterial anatomy poses a challenge for surgeon during pancreaticoduodenectomy (PD). These anomalies are best picked up on pre-operative imaging to avoid inadvertent injury to the aberrant vasculature resulting in liver ischaemia or biliary-enteric anastomotic failure. We present our experience of dealing with aberrant hepatic vessels during PD.

Methods: Patients with aberrant hepatic vasculature who underwent PD between September 2014 and August 2015 were included in the study. We used artery first technique for dissection in cases identified on pre-operative imaging. Aberrations were classed according to Hiatt classification.

Results: A total of 23 PD were performed with aberrant arterial anatomy in 10 (43\%) cases. These vessels were recognised and preserved in nine cases. In one patient, the replaced right hepatic artery (RRHA) arising from superior mesenteric artery (SMA) was coursing through pancreatic parenchyma needing resection and reconstruction with uneventful post-operative recovery. We also identified one RRHA arising from SMA coursing lateral to common bile duct and entering liver parenchyma in gallbladder fossa.
\end{abstract}

Conclusion: Aberrant hepatic arterial anomalies are common and should ideally be picked up by pre-operative imaging. It is possible to preserve these vessels in most cases with careful surgical dissection using artery first technique. Surgeons performing PD should be well versed with the aberrant vascular anatomy to minimise any inadvertent damage.

Key words: Aberrant hepatic artery, artery first technique, pancreatic oduodenectomy

\section{Introduction}

Pancreaticoduodenectomy (PD) offers the only chance of curative treatment of resectable pancreatic cancer. ${ }^{[1]}$ Although the mortality for this complex procedure has improved significantly over the past few decades, the morbidity still remains very high with post-pancreatectomy haemorrhage and anastomotic leakage being the most significant complications. ${ }^{[2]}$ Aberrant hepatic arterial anatomy is encountered in approximately $25 \%$ of patients. ${ }^{[3]}$ These anomalies are best picked up on preoperative imaging to avoid inadvertent injury to the

Correspondence: Dr. Faisal Hanif, Department of Surgical

Oncology, Shaukat Khanum Memorial Cancer Hospital and Research Centre, Lahore, Pakistan.

Email: faisalhanif@skm.org.pk aberrant vasculature..$^{[4]}$ This may result in haemorrhage, inadequate perfusion to liver or bile duct with subsequent anastomotic failure. ${ }^{[5]}$

Aberrant right hepatic artery is the most common anomaly found in the hepatic vasculature. ${ }^{[3,6]}$ Michels $^{[7]}$ described vascular anatomic variations in 1966 which were classified into 10 types which were later modified into six types by Hiatt et al. ${ }^{[3]}$ [Table 1]. According to Hiatt classification, ${ }^{[3]}$ the replaced right hepatic artery (RRHA) arising from the superior mesenteric artery (SMA) occurs in $10.6 \%$ of cases. This presents problem with possibility of haemorrhage, ischaemia to the liver or bile duct or inadvertent damage to the adventitia of the vessel predisposing the patient to subsequent haemorrhagic complications. ${ }^{[4]}$ 
Table 1: Classification of hepatic arterial variations described by Hiatt et al. ${ }^{[3]}$

\begin{tabular}{l|l|c}
\hline Variation & Hiatt type & Incidence (\%) \\
\hline Normal vascular pattern & Type 1 & 75.7 \\
\hline $\begin{array}{l}\text { Aberrant left hepatic ar- } \\
\text { tery from the left gastric } \\
\text { artery }\end{array}$ & Type 2 & 9.7 \\
\hline $\begin{array}{l}\text { Aberrant right hepatic } \\
\text { artery from superior } \\
\text { mesenteric artery }\end{array}$ & Type 3 & 10.6 \\
\hline $\begin{array}{l}\text { Aberrant RHA and aber- } \\
\text { rant LHA }\end{array}$ & Type 4 & 2.3 \\
\hline $\begin{array}{l}\text { Common hepatic artery } \\
\text { from superior mesenteric } \\
\text { artery }\end{array}$ & Type 5 & 1.5 \\
\hline $\begin{array}{l}\text { Common hepatic artery } \\
\text { from aorta }\end{array}$ & Type 6 & 0.2 \\
\hline
\end{tabular}

RHA: Right hepatic artery, LHA: Left hepatic artery

An aberrant vessel, especially a replaced right hepatic vessel arising from SMA, may course posterior to the pancreatic head or portal vein and thus may lie in the area of dissection. This situation places the aberrant vessel at risk of damage during resection. The aberrant vessel can be preserved in most of the circumstances by meticulous dissection; however, it may require ligation in up to $20 \%$ of cases.$^{[8]}$ We report our institutional experience of dealing with aberrant arterial anatomy encountered during PD.

\section{Methods}

Patients undergoing PD at Shaukat Khanum Memorial Cancer Hospital and Research Centre, Lahore, Pakistan, between September 2014 and August 2015 were included in the study. Arterial anomalies were identified on preoperative cross-sectional imaging which included biphasic computed tomography scan with pancreatic protocol in majority of the patients. Operative details were recorded regarding intraoperative identification management of aberrant vascular anatomy.

Artery first dissection was performed for aberrant vessels arising from SMA or for an aberrant vessel coursing caudal or lateral to the portal vein. Standard dissection for PD was performed for normal vascular anatomy or for other aberrant variations of hepatic vasculature.

\section{Results}

During the study period of 1 year, a total of 23 patients underwent PD Normal vascular anatomy was observed in $13(56.5 \%)$ cases. Aberrant hepatic arterial anatomy was encountered in $10(43.4 \%)$ cases. Details of aberrant vascular anatomy and the management are described in Table 2. The most common aberration was a replaced RRHA arising from the SMA which was seen in $6(26 \%)$ cases followed by common hepatic artery arising from the SMA in $2(8.7 \%)$ cases. In both these types of anomalies, artery first dissection was performed as the aberrant vessels were coursing through the area of proposed dissection. In one case, the RRHA was coursing through pancreatic parenchyma and had to be sacrificed for oncological reasons. This vessel was reconstructed by end-to-end anastomosis. Post-operative scans of this patient showed patency of the reconstructed vessel.

There were 13 (56.5\%) patients with Type 1 (normal) arterial anatomy according to Hiatt classification, one (4.4\%) with Type 2 and Type 6 both. We did not encounter Hiatt Type 4 vascular anomalies in our study population. All apart from one of these aberrant vessels were identified and preserved during dissection. In only one case, resection and reconstruction of aberrant vessel were performed for oncological clearance. We also encountered RRHA arising from SMA coursing lateral to common bile duct (CBD) and entering liver parenchyma in gallbladder fossa in one patient. This anomaly is not described in literature and is at high risk of injury during cholecystectomy. One patient had Type 3 anomaly (RRHA originating from SMA), but the course of artery was further abnormal as it courses lateral to CBD and entered in gallbladder fossa. This anomaly is not reported in literature and carries high risk of damage in cholecystectomies.

\section{Discussion}

We present our institutional experience of aberrant hepatic arterial anatomy encountered during PD. This is the first description of variant hepatic vascular anatomy from Pakistan and represents data from our population. We encountered anomalous vessels in $43 \%$ of cases undergoing PD. This proportion is significantly higher compared to previously describe international literature. Aberrant vessels were seen in $24.3 \%$ of patients in Hiatt's landmark paper on hepatic arterial anatomy. ${ }^{[3]}$ Till date, 
Table 2: Hepatic artery aberrations according to Hiatt classification and their intraoperative management

\begin{tabular}{l|c|c|c}
\hline Variation & Hiatt type & Number (\%) & Management \\
\hline Normal vascular pattern & 1 & $13(56.5)$ & SD \\
\hline Replaced RHA from SMA & 3 & $06(26)$ & $\mathrm{AF}$ \\
\hline CHA from SMA & 5 & $02(8.7)$ & $\mathrm{AF}$ \\
\hline Accessory LHA from LGA & 2 & $01(4.4)$ & $\mathrm{SD}$ \\
\hline CHA from aorta & 6 & $01(4.4)$ & $\mathrm{SD}$ \\
\hline
\end{tabular}

RHA: Right hepatic artery, SMA: Superior mesenteric artery, CHA: Common hepatic artery, LHA: Left hepatic artery, LGA: Left gastric artery, SD: Standard dissection, AF: Artery first dissection; aberrant artery slung followed by standard dissection
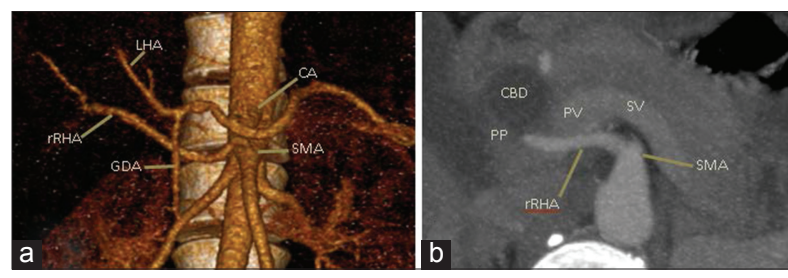

Figure 1: Reconstructed image (a) showing the replaced right hepatic artery (RRHA) arising from superior mesenteric artery (SMA). (b) Axial sections showing the artery traversing the pancreatic parenchyma lateral to the portal vein and the common bile duct (CBD). RRHA, LHA - left hepatic artery, GDA - gastroduodenal artery, CA - celiac axis, SMA, CBD, $\mathrm{PV}$ - portal vein, SV - splenic vein, PP - pancreatic parenchyma

no local data are published on hepatic vascular anomaly. More recent literature looking at the pattern of aberrant hepatic vasculature also shows an incidence of aberrant hepatic vasculature in $16-19 \%{ }^{[2,5,8]}$ We need larger sample to evaluate more accurate incidence of hepatic arterial anomaly in our patients as we lack local data.

The significance of identifying vascular pattern lies in planning dissection precluding inadvertent ligation of hepatic artery while performing PD as the anomalous artery may course through the proposed area of dissection. We performed artery first dissection by identifying the aberrant vessel before dissection and transection of the pancreas. The identified aberrant vessel was slung in a vascular loop to ensure its preservation during the dissection. Artery first dissection was performed in all cases where the aberrant vessel was arising from the SMA since the vessel was coursing through the proposed area of dissection and would thus be at risk of damage during this dissection.

In cases where the aberrant vessel was not coursing through the area of proposed dissection (Hiatt Type 2 or
Type 6 arterial anomaly), standard dissection technique was used as the vessel was not at a high risk of damage during dissection. In one patient, the aberrant vessel had to be sacrificed as it was coursing through the pancreatic parenchyma and required resection for oncological reasons. This vessel was resected and reconstruction by end-to-end anastomosis was performed [Figure 1].

\section{Conclusion}

This discrepancy in incidence of vascular anomalies in our population suggests the need for a more conscious effort to identify such variations on pre-operative imaging. Failure to do so may result in inadvertent damage to these vessels with significant increase in the morbidity following this complex procedure. Majority of the aberrant vessels can be preserved by meticulous dissection, and in our experience, artery first technique is a suitable option to achieve this objective.

\section{Conflict of Interest}

The authors declare that they have no conflict of interest.

\section{References}

1. Ducreux M, Cuhna AS, Caramella C, et al. Cancer of the pancreas: ESMO clinical practice guidelines for diagnosis, treatment and follow-up. Ann Oncol 2015;26 Suppl 5:56-68.

2. Eshuis WJ, Loohuis KM, Busch OR, et al. Influence of aberrant right hepatic artery on perioperative course and longterm survival after pancreatoduodenectomy. HPB (Oxford) 2011;13:161-7.

3. Hiatt JR, Gabbay J, Busuttil RW. Surgical anatomy of the hepatic arteries in 1000 cases. Ann Surg 1994;220:50-2.

4. Christians KK, Tsai S, Tolat PP, et al. Critical steps for pancreaticoduodenectomy in the setting of pancreatic adenocarcinoma. J Surg Oncol 2013;107:33-8. 
5. Stauffer JA, Bridges MD, Turan N, et al. Aberrant right hepatic arterial anatomy and pancreaticoduodenectomy: Recognition, prevalence and management. HPB (Oxford) 2009;11:161-5.

6. Allendorf JD, Bellemare S. Reconstruction of the replaced right hepatic artery at the time of pancreaticoduodenectomy. J Gastrointest Surg 2009;13:555-7.
7. Michels NA. Newer anatomy of the liver and its variant blood supply and collateral circulation. Am J Surg 1966;112:337-47.

8. Rammohan A, Palaniappan R, Pitchaimuthu A, et al. Implications of the presence of an aberrant right hepatic artery in patients undergoing pancreaticoduodenectomy. World J Gastrointest Surg 2014;6:9-13. 